\title{
Design and Implementation of Integrated Assembly Object Model for Intelligent Virtual Assembly Planning*
}

\author{
Jing Fan, Yang Ye, and Jia-Mei Cai \\ College of Information Engineering, Zhejiang University of Technology \\ Zhaohui Liuqu, Hangzhou, 310014, P.R. China \\ fanjing@zjut.edu.cn
}

\begin{abstract}
Based on the analysis of the virtual assembly planning, this paper presents a new assembly planning method, which is named Intelligent Virtual Assembly Planning. The architecture of the IVAP system consists of four main components: Intelligent Assembly Planning Environment, Virtual Assembly Planning Environment, Data/Knowledge Acquisition Interface, and Inner Interactive Interface. The integrated assembly object model used in the system is described in detail in the paper.
\end{abstract}

\section{Introduction}

The rapid development of Virtual Environment provides a new tool to assembly planning of products. After getting data of the virtual components, and applying Virtual Environment as interactive interface to help to complete the task of assembly planning, virtual prototype of the product can be obtained. With the support of Virtual Environment and computer system, the engineer can manipulate the virtual components effectively and easily to create, simulate and evaluate assembly sequence. The way working in Virtual Environment is similar to the way of working in the real environment, and is easily accepted by the user.

The research on virtual assembly has developed quickly in the world. But it still has many limitations. For example, the user in Virtual Environment can not work for a long time, because his eyes wearing HMD easily feel tired; the delay between assembly operation and display result affects the precision of assembly; and the technique of collision detection on line still need to be improved. On the analysis of the technology of Computer Aided Assembly Planning, a new method of virtual assembly planning is proposed, which applies the formalized assembly knowledge of expert that is obtained using the technology of artificial intelligence in the virtual assembly, and creates Integrated Assembly Model to represent different kinds of assembly data and knowledge, together with reasoning strategy, to implement the intelligent virtual assembly planning.

\footnotetext{
* The research work in this paper is supported by the project of Zhejiang Natural Science Foundation of China (No. 602092).
} 
This paper introduces the main idea of intelligent virtual assembly planning, and focuses on the Integrated Assembly Object Model used in intelligent virtual assembly planning.

\section{Related Research}

The first research of assembly model was done by Lieberman and Welsey in 1977. They have used graph structure to describe the information of assembly, in which node represents the part, and arc represents the relation between parts [1]. After that there are a lot of research work have been done in this area, such as the liaison graph proposed by Bourjault [2], the AND/OR graph used by Homem De Mello and Sanderson [3], the assembly constraints graph described by J. Wolter [4], and the virtual link designed by Lee and Gossard [5], etc. These assembly models are used to generate the assembly sequence automatically. But the common limitation of these models is that they can not express the assembly knowledge efficiently.

The assembly models used in virtual environment are usually based on the geometry model. They can express the geometry features of the parts effectively, but ignore other important non-geometry information like the physical properties and assembly semantics [6],[7]. Using this kind of assembly models, the assembly knowledge and experiences from the planning experts is difficult to be represented and utilized in a formalized way.

\section{Intelligent Virtual Assembly Planning}

The main idea of the intelligent virtual assembly planning is as follows: using the new interactive tool provided by virtual environment in the assembly planning, and applying the techniques of AI to support the virtual assembly planning, to improve the suitability and efficiency of the assembly planning to the complex assembly objects.

The architecture of intelligent virtual assembly planning system is shown in Fig. 1. There are four main parts in the system:

- Intelligent Assembly Planning Environment. It contains the Integrated Data/ Knowledge Model, Intelligent Planner and Evaluator. Integrated Data/Knowledge Model consists of integrated assembly object model, scene model and interaction model. The integrated assembly object model is the emphasis of this paper, which is used to express the assembly objects.

- Virtual Assembly Planning Environment. It contains Simulation Module, State Control Module, and Input/Output Module, etc.

- Data and Knowledge Acquisition Interface. It provides the interface to input data from other systems and get assembly knowledge from the assembly planning experts. The assembly data and knowledge are transformed to construct the Integrated Assembly Model, and define assembly rules. 
- Inner Interactive Interface. It is used to implement the communication and coordination between Intelligent Assembly Planning Environment and Virtual Assembly Planning Environment. The assembly data and knowledge are exchanged and shared through the Inner Interactive Interface.

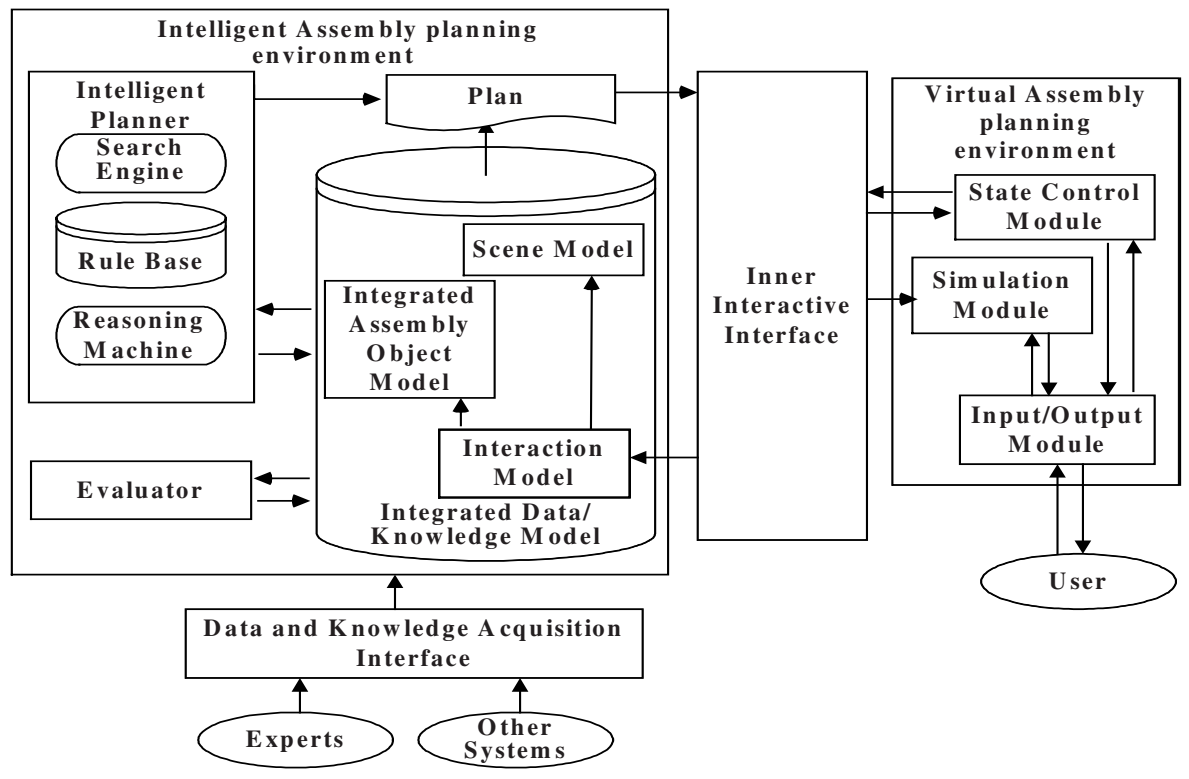

Fig. 1. Architecture of Intelligent Virtual Assembly Planning System

\section{Integrated Assembly Object Model}

Since the assembly models that have been proposed in the past time can not efficiently express the complex assembly objects, especially in the virtual assembly environment for the virtual assembly planning, we present the integrated assembly object model to express the assembly data and knowledge of the virtual components.

The integrated assembly object model contains data model, constraint model, and semantic model.

- Data model expresses the basic information of virtual assembly objects such as geometry features and physical properties in a unique form.

- Constraint model describes the constraints of virtual assembly objects, which mean the relations between the objects that cannot be expressed in the data model. All the assembly constraints are expressed by predicate.

- Semantic model describes the semantic of a group of assembly objects, including the information of assembly process and the operations of the assembly objects. 


\subsection{Data Model}

Virtual assembly objects is clustered and described in a unique form, which is called Virtual Assembly Concept (VAC). Formalized expression of VAC is as the follows:

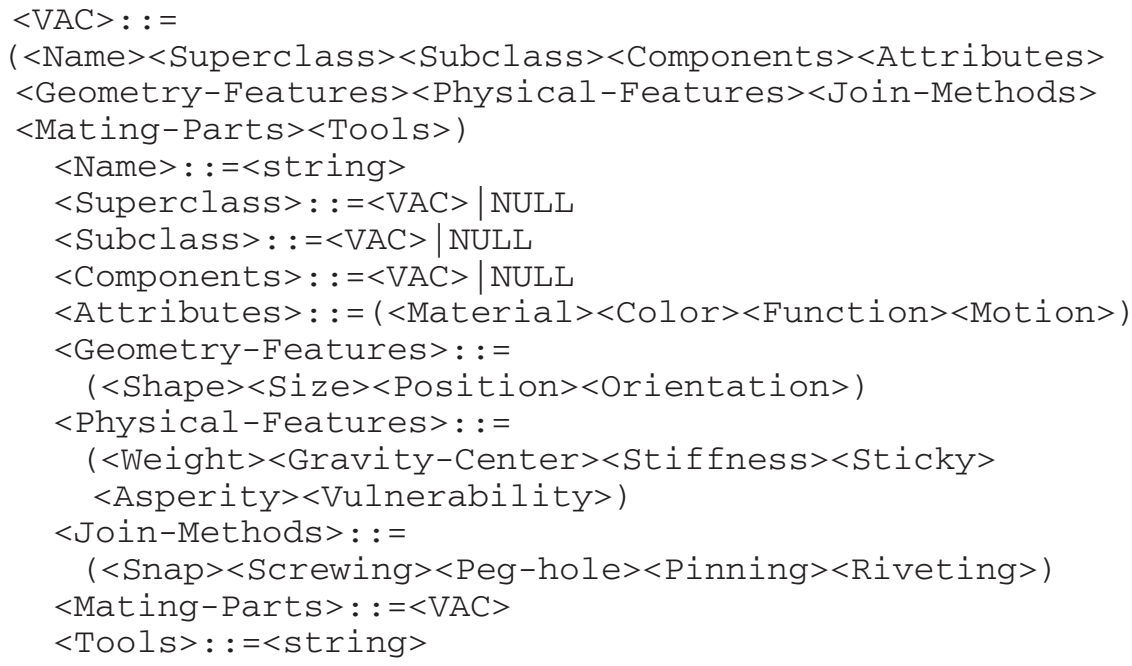

\subsection{Constraint Model}

The constraints in the constraint model are classified as two kinds, Low-level constraints and High-level constraints. Low-level constraints are established on the basis of data model while High-level constraints are based on the Low-level constraints.

Low-level constraints are composed of Low-level geometry constraints and Lowlevel physical constraints. Low-level geometry constraints represent geometry of the assembly objects, such as position, orientation, mate, co-axis, insertion, etc. Lowlevel physical constraints represent the force effects between the objects.

High-level constraints represent the abstract information of the relations between the objects. It includes: High-level geometry constraints, High-level physical constraints, topology constraints, and precedence constraints, etc. User defined constraints, like cost constraint and time constraint, which are named soft constraints, are also included in the High-level constraints.

\subsection{Integrated Semantic Model}

The assembly semantic of the virtual assembly objects is expressed by the integrated semantic model (ISM), which is established in a higher level to describe the dynamic information between objects, such as assembly operations, sequence and process.

Assembly process information describes the assembly process of the group of virtual object according to the semantic. It includes transformation from initial state to 
final state. The changes of the states are made by the operation steps. Each step is corresponding to a list of assembly operations.

Assembly sequence information describes the precedence of the assembly operations to the objects.

Assembly operation describes the assembly operations on the objects, which consists of basic meta operations, as translation and rotation.

Formalized expression of the ISM is a quintuple.

$\mathrm{ISM}=<\mathrm{E}, \mathrm{O}, \mathrm{P}_{\text {info, }}, \mathrm{S}_{\text {info, }}, \mathrm{O}_{\text {info }}>$

Where $E$ is the set of the assembly objects, $O$ is the set of the assembly operations, $P_{\text {info }}$ stores the process information, $S_{\text {info }}$ presents the assembly sequence information and $O_{\text {info }}$ represents the information of assembly operation.

$$
E=\left\{P_{i} \mid i=1, \cdots, n\right\}
$$

There are $n$ assembly objects $P_{i}$.

$$
O=\left\{O_{j}\left(P_{i}\right) \mid i=1, \cdots n_{i} j=1,2\right\}
$$

There are $n$ assembly objects, each have two meta-operations, translation $O_{1}$ and rotation $\mathrm{O}_{2}$. There are $2 n$ operations totally.

$$
\mathrm{P}_{\text {info }}=\left\{\mathrm{S}_{\mathrm{p}-1}, \mathrm{STEP}_{\mathrm{p}} \mid \mathrm{p}=1, \cdots, t\right\}
$$

There are $t+1$ states and $t$ steps. $S_{o}$ is the initial state and $S_{t}$ is the final state. $S_{p-1}$ is transformed to state $S_{p}$ with Step $S T E P_{p}$. Each step is composed of a list of assembly operations.

$$
S_{\text {info }}=\{S E, S A\}
$$

$S E$ is the sequence between the assembly objects.

$S A$ is the sequence between the operations.

$$
\begin{aligned}
& O_{\text {info }}=\left\{A_{k} \mid k=1, \cdots, m\right\} \\
& A_{k} \in\left\{O_{j}\left(P_{i}\right) \mid i=1, \cdots, n ; j=1, \cdots, 2\right\}
\end{aligned}
$$

There are $m$ operations used in the assembly process.

\section{The Implementation of the Model}

\subsection{Data Model}

The virtual components are described in VRML2.0.

The description of the virtual components is constructed with two parts: nodes and features. Each node represents one kind of virtual components.

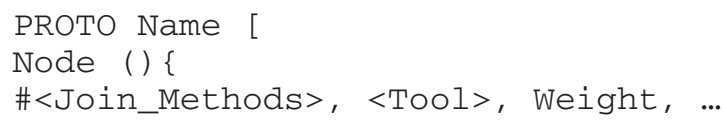




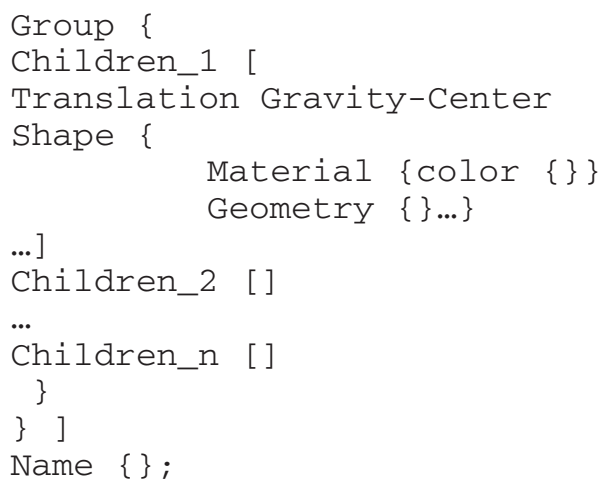

The features of the virtual component used in the assembly are extracted from node. Different nodes have different features. The basic geometry feature is represented in the following structure:

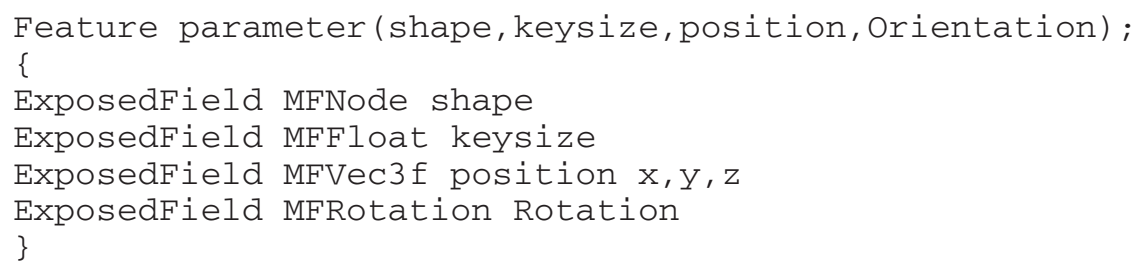

For example, the basic geometry features of the part bolt are showed as follows:

Bolt (Cylinder, <radius, height>, <x, y, z>, rotation)

\subsection{Constraint Model}

Feature parameter can be transformed to lower geometry constraints automatically. After all the components are created in the VRML, the data of the components are transformed to constraints and stored in the Constraint Model.

The following are some Low-level constraints used popularly.

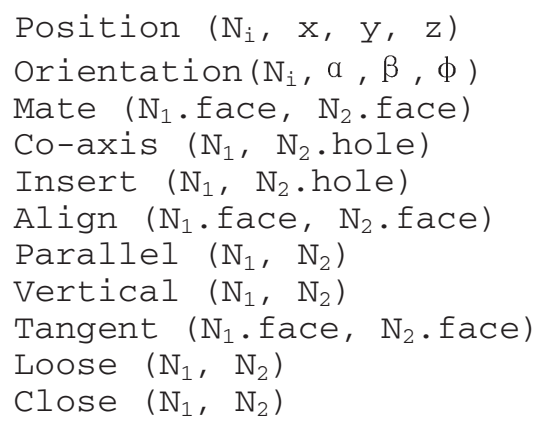

High-level constraints are established on the Low-level constraints. Here are some High-level constraints. 
Interfering $\left(\mathrm{N}_{1}, \mathrm{~N}_{2}\right)$

Connect $\left(\mathrm{N}_{1}, \mathrm{~N}_{2}\right)$

Proceed $\left(\mathrm{N}_{1}, \mathrm{~N}_{2}\right)$

\subsection{Semantic Base}

The components that are contributed to one specialized assembly function are collected together to construct the assembly semantic.

The Semantic of "Part1 and part2 are jointed by bolt" is:

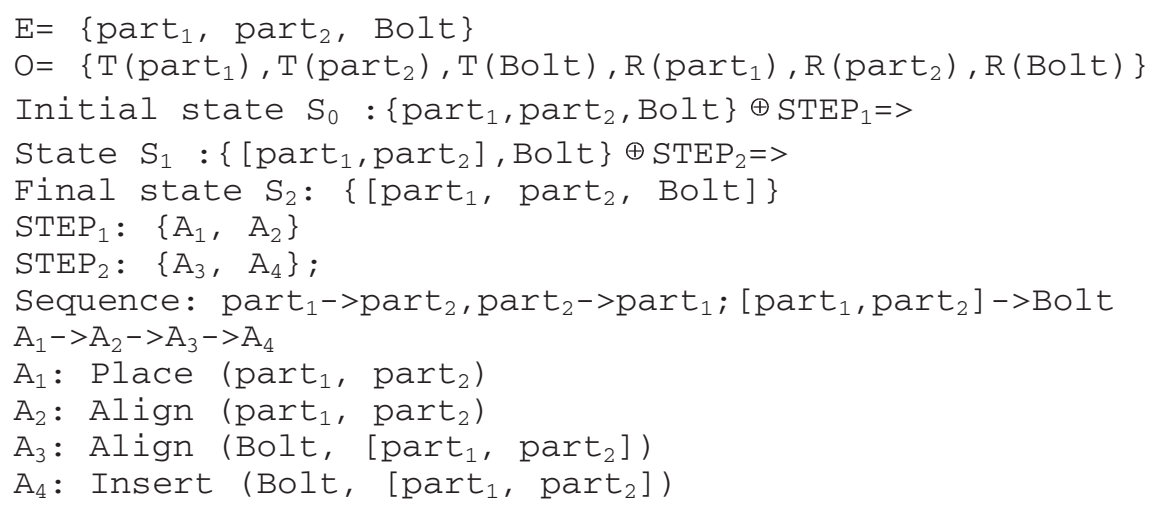

\subsection{Example}

In the example there are eight kinds of components, Axle, Base, Bolt, Bushing, L_Bracket, Pulley, Washer and Key (see Figure2), which are expressed by eight nodes in VRML And their geometry features are gotten by function call through the VRML In/Out event interface.

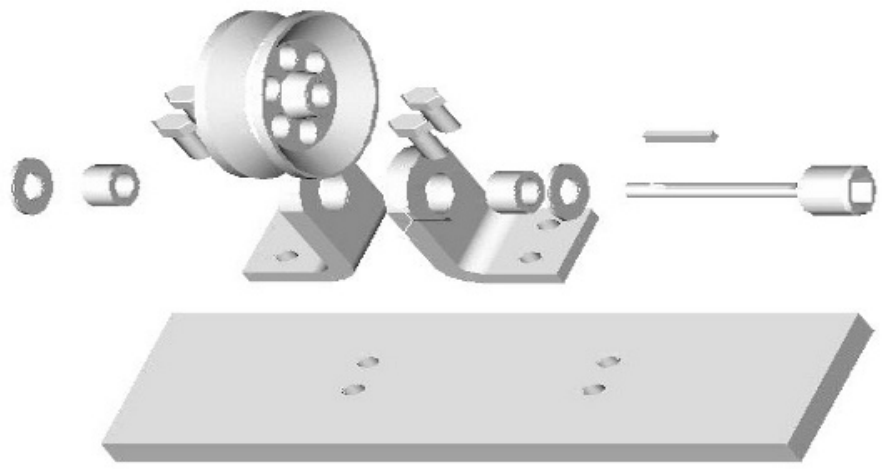

Fig. 2. Example 
There are 15 constraints and three semantics totally obtained from the data model. And here are the three assembly semantics.

1.'Part Base and Part L_Bracket are jointed by Part Bolt"

2.'Part L_Bracket, Part Pulley and Part Axle are jointed"

3."Part Key is placed in the slot on Part Axle"

\section{Summary}

Although the mechanical parts can be created in the 3D model easily, the relations between parts cannot be described effectively. The integrated assembly object model can be used to express the data and knowledge of the virtual assembly objects in different level for assembly planning. The establishment of the powerful assembly model will help to simplify and improve virtual assembly planning.

\section{References}

1. Lieberman L. I., Wesley M. A.: AUTOPASS: An Automatic Programming System for Computer Controlled Mechanical Assembly, IBM Journal of Research and Development, 21(4), (1977) 321-333

2. Bourjault A.: Contributionune approche methodologique de l'assemblage automatise: elaboration automatique des sequences operatiores, Thesis d'Etat Universite de FrancheComte, Besancon, France (1984)

3. Homem de Mello L., Sanderson A.: And/Or Graph Representation of Assembly Plans, IEEE Transactions on Robotics and Automation, 6(2), (1990) 188-199

4. Wolter J.: On The Automatic Generation Of Assembly Plans, Computer-Aided Mechanical Assembly Planning, Kluwer Academic Publishers (1991)

5. Lee K., Gossard D. C.: A Hierarchical Data Structures for Representing Assemblies, part I., Computer Aided Design, 17(1), (1985) 15-19

6. Bullinger H.J., Richter M. and Seidel K.A.: Virtual Assembly Planning, Human Factors and Ergonomics in Manufacturing, 10(3), (2000) 331-341

7. Dewar R., Carpenter I. D., Ritchie J. M., and Simmons J. E. L: Assembly Planning in a Virtual Environment, Proceedings of PICMET '97 Portland USA (1997) 664-667 